\title{
Mitochondrial gene sequence variants in children with severe malaria anaemia with or without lactic acidosis: a case control study
}

Casey Fowler ${ }^{1}$, Christine Cserti-Gazdewich², Aggrey Dhabangi ${ }^{3}$, Charles Musoke ${ }^{4}$, Himanshu Sharma ${ }^{1}$, Sami S. Amr ${ }^{1}$ and Walter Dzik ${ }^{5^{*}}$ (i)

\begin{abstract}
Background: Evolutionary pressure by Plasmodium falciparum malaria is known to have favoured a large number of human gene adaptations, but there is surprisingly little investigation of the effect of malaria on human mitochondrial sequence variation. Plasmodium falciparum infection can cause severe malaria anaemia (SMA) with insufficient tissue oxygenation, lactic acidosis and death. Despite equal degrees of severe anaemia, some individuals develop lactic acidosis while others do not. A case-control study design was used to investigate whether differences in host mitochondrial gene sequences were associated with lactic acidosis in SMA. Full mitochondrial sequences were obtained from 36 subjects with SMA complicated by lactic acidosis and 37 subjects with SMA without lactic acidosis. The two groups were matched for age, sex, and degree of anaemia.
\end{abstract}

Results: Compared with the reference sequence, a median of 60 nucleotide variants per individual (interquartile range 4-91) was found, with an average frequency of 3.97 variants per 1000 nucleotides. The frequency and distribution of non-synonymous DNA variants in genes associated with oxidative phosphorylation were not statistically different between the two groups. Non-synonymous variants predicted to have the most disruptive effect on proteins responsible for oxidative phosphorylation were present at a similar frequency in both groups.

Conclusions: Lactic acidosis in SMA does not appear to be consistently associated with the high prevalence of any mitochondrial gene variant.

Keywords: Mitochondria, Malaria, Lactic acidosis, Anemia, Tissue oxygenation, DNA sequence

\section{Background}

Malaria results in the death of hundreds of thousands of children each year [1]. Genetic adaptations affecting host susceptibility play a major role in survival from malaria $[2,3]$. Because most deaths from Plasmodium falciparum malaria occur in children prior to puberty, malaria has been recognized as "the strongest known force for evolutionary selection in the recent history of the human

\footnotetext{
*Correspondence: sdzik@mgh.harvard.edu

${ }^{5}$ Department of Pathology and Medicine, Massachusetts General Hospital, Boston, MA, USA

Full list of author information is available at the end of the article
}

genome" [4]. Severe malaria anaemia (SMA) is a wellrecognized syndrome of falciparum malaria $[3,5]$. The concentration of haemoglobin in children with SMA is below $5 \mathrm{~g} / \mathrm{dL}$ and patients exhibit weakness, prostration, tachycardia and respiratory distress. This depth of anaemia can result in insufficient delivery of oxygen to tissues with resulting systemic lactic acidosis [5]. Lactic acidosis in malaria, if not corrected, is highly correlated with death [6].

Approximately $50 \%$ of Ugandan children with SMA have elevated blood lactate levels [3, 5, 6]. Blood lactate production results from host metabolism and has been found not to correlate with parasite burden. Although 
it has been shown that lactic acidosis can result from severe anaemia with inadequate tissue oxygenation, and that lactic acidosis is relieved by transfusion of red blood cells with restoration of tissue oxygenation [5], there is no recognized explanation for the observation that some children develop lactic acidosis with SMA while others do not. Because oxygen-dependent bioenergetics occurs within mitochondria and relies in part on proteins encoded by mitochondrial genes, this study was designed to explore whether genetic differences in host mitochondria might account for differences in tolerance to the decreased tissue oxygenation that accompanies SMA. To investigate whether or not mitochondrial sequence variation might be associated with the development of lactic acidosis in severe anaemia, a matched case-control study among children with SMA was performed to compare the entire mitochondrial genome among severely anemic children with and without lactic acidosis.

\section{Methods}

\section{Patient selection}

Patient blood samples for mitochondrial DNA sequencing were collected during the course of two prior studies $[3,5]$. All samples were obtained from children aged 6 months to 5 years presenting for care to the paediatric acute care unit of Mulago Hospital in Kampala, Uganda. The hospital serves an indigent local community population which is ancestrally homogeneous with over $75 \%$ of mothers belonging to one of three tribes (Muganda $63 \%$, Musoga $8 \%$, and Munyankole $9 \%$ ). All samples came from children with confirmed P. falciparum infection and with haemoglobin values $\leq 5 \mathrm{~g} / \mathrm{dL}$.

Blood lactate and haemoglobin levels were measured using point-of-care devices (LactatePro, Arkray; Hemocue 201) at the time of sample collection and prior to blood transfusion. Samples were frozen immediately after phlebotomy and stored in cryovials at $-20{ }^{\circ} \mathrm{C}$ or colder prior to testing. All samples were collected and stored with informed consent of the parent or guardian. Approval for sample collection, material transfer, and DNA sequencing was provided by the research ethics committee of Makerere University in Kampala, Uganda and by the Uganda National Council of Science and Technology.

From the above archive of frozen samples, two groups were selected based on the blood lactate level measured at the time of original phlebotomy: one group $(n=50)$ with blood lactate levels $>10 \mathrm{mM}$ and one group with blood lactate levels $<5 \mathrm{mM}(\mathrm{n}=50)$. Samples from the two groups were deliberately matched for patient age, gender, and haemoglobin concentration. Only patients who tested negative for the presence of haemoglobin $\mathrm{S}$ were included.

\section{DNA extraction}

For each patient, DNA was purified from a frozen blood sample using the Autopure LS instrument following the manufacturer's protocol (AutoGen, MA, USA). An additional purification was performed using the ZR-96 DNA Clean \& Concentrator-5 kit (Zymo Research, CA, USA) to reduce heparin contamination. Purified DNA samples were then quantified using the Quant-iT PicoGreen dsDNA assay kit (Invitrogen, CA, USA) and measurements were made on an ultraviolet spectrophotometer (Gemini XPS).

\section{DNA library preparation}

Library preparation for each DNA sample was performed by long-range PCR followed by Nextera XT (Illumina, CA, USA) as described in the Human mtDNA Genome protocol for the Illumina Sequencing Platform [7]. Briefly, two long PCR amplicons were generated using two sets of primers (MTL-F1 and MTL-R1, MTL-F2, and MTL-R2) designed to encompass the entire human mitochondrial genome. The first set of primers generated a $9.1 \mathrm{~kb}$ fragment using primers MTL-F1 (5'-AAA GCA CAT ACC AAG GCC AC-3') and MTL-F2 (5'-TTG GCT CTC CTT GCA AAG TT-3'). The second set of primers generated an $11.2 \mathrm{~kb}$ fragment using primers MTL-F2 (5'-TAT CCG CCA TCC CAT ACA TT-3') and MTL-R2 (5'-AAT GTT GAG CCG TAG ATG CC-3'). For each patient DNA, two long-range PCR amplifications were performed using the TaKaRa LA TaqDNA Polymerase kit with a DNA input of $20 \mathrm{ng}$. Post-PCR products were quantified as described above and fragment sizes were verified using the DNA 12000 Bioanalyzer kit (Agilent, CA, USA).

The two amplicons for each patient were pooled and libraries were generated using the NexteraXT protocol (Illumina, CA, USA) automated on the Sciclone (Caliper/Perkin Elmer) with a DNA input of $1 \mathrm{ng}$. The NexteraXT library prep kit is a tagmentation process that fragments and adds adaptors to those fragments using an engineered transposome. The tagmented DNA is then amplified using a limited-cycle PCR program which adds Illumina adapters and unique indexes for each patient amplicon pool. After PCR, samples are purified using Ampure XP beads (Beckman Coulter, MA, USA).

Final libraries were quantified and fragment sizes were determined using High Sensitive D1000 Screentapes on the TapeStation instrument (Agilent, CA, USA). The expected size of final libraries is in the range of 500-1000 bp. Accurate measurement of sequence ready libraries was performed using the KAPA Library Quantification Kit (KAPA Biosystems, MA, USA) and run on 
the 7500 Fast Real-Time PCR System (Applied Biosystems, CA, USA).

\section{Sequencing}

The uniquely indexed patient libraries were pooled at equimolar concentrations based upon KAPA assay measurements, and the final pool was loaded at $10 \mathrm{pM}$ (with 5\% PhiX spike-in) on the Miseq instrument (Illumina, CA, USA) using a 600 cycle v3 Miseq kit (Illumina) to generate 300 bp paired end sequenced reads.

\section{Variant calling and coverage}

Raw sequencing data were analysed using the BaseSpace mtDNA Variant Processor App (Illumina), a commercially available web-based analysis pipeline. Briefly, this tool performs alignment and variant calling of samples against a reference mitochondrial DNA (mtDNA) genome, generating BAM alignment files and variant call files (VCF) for each of the samples. The analysis was restricted to samples with $>90 \%$ coverage of the entire mitochondrial sequence at $10 \times$ reads or greater. Additional information on software components of this analysis pipeline can be found online (https://support.illum ina.com/downloads/basespace-mtdna-variant-processorapp.html).

\section{Variant annotations}

Variant annotations were performed using MSeqDR: the Mitochondrial Disease Sequence Data Resource Consortium's tool called MvTool and VariantOneStop (https $: / /$ mseqdr.org/). Briefly, VCF files were processed to the required format by using python scripts and run through mvTool to get variant annotations in csv format. mvTool converts dozens of mtDNA variant formats into a standard revised Cambridge Reference Sequence (rCRS)-based HGVS format.

In addition, annotations generated by VariantOneStop include multiple-population frequencies from Mitomap and HmtDB, as well as annotations from Ensembl VEP, Mutalyzer, ClinVar, and from the MSeqDR database. Functional predictions for missense variants were curated from MitImpact2 database which provides pre-computed pathogenicity predictions for mitochondrial variants (http://mitimpact.css-mende l.it/) and includes predictions from MToolBox, PolyPhen2, MutationVariant Taster, and SIFT tools.

Haplogroup assignment for each individual was performed using the mtDNA-Server analysis software (https://mtdna-server.uibk.ac.at/index.html), which utilizes the HaploGrep tool to determine haplogroup of mtDNA profiles (http://haplogrep.uibk.ac.at/). Bam files for each sample were uploaded directly into mtDNA-Server for this analysis.

\section{Statistical analysis}

For each group the median number of variants per person relative to the Cambridge Reference Sequence was compared using the Wilcoxon test; and the average number of variants per 1000 nucleotides was compared using the Student's t-test. The Fisher's test was used to compare the frequency of disruptive non-synonymous variants in the NADH dehydrogenase gene cluster. An alpha value of 0.05 was used to assess statistical significance. No corrections were made for multiple comparisons.

\section{Results}

From the original set of 100 archived samples, 7 were eliminated due to low DNA isolation and an additional 20 were eliminated due to low coverage after sequencing, leaving 73 subjects (36 with high blood lactate levels and 37 with normal lactates) for final analysis. See "Methods" for details. Clinical characteristics of the comparison groups are shown in Table 1. All patients had active P. falciparum malaria and met standard criteria for SMA. The two groups were well-matched for age and sex. Of particular importance, the two groups were matched for haemoglobin concentration and oxygen saturation, determinants of oxygen delivery, but had blood lactate levels that differed by more than fivefold.

Table 1 Clinical characteristics of the high lactate and normal lactate groups

\begin{tabular}{lll}
\hline & High lactate, $\mathbf{n = 3 6}$ & Normal lactate, $\mathbf{n = 3 7}$ \\
\hline Median (IQR) lactate, $\mathrm{mM}$ & $14.4(13.6-15.3)$ & $2.7(1.9-3.3)$ \\
Median (IQR) age, years & $2.3(1.5-3.8)$ & $2.5(1.7-4.2)$ \\
Gender (female:male) & $16: 20$ & $14: 23$ \\
Median (IQR) haemoglobin, g/dL & $2.8(2.6-3.4)$ & $3.0(2.8-3.7)$ \\
Mean (SD) $\mathrm{O}_{2}$-saturation, \% & $96.8(4.1)$ & $97.5(2.4)$ \\
Mean (SD) log parasitaemia & $4.66(0.79)$ & $4.28(1.07)$ \\
\hline
\end{tabular}

${ }^{a}$ The $\log _{10}$ of the number of parasitized red cells per $\mu \mathrm{L}$ 


\section{Haplogroup assignments}

Mitochondrial haplogroup assignments were made as described in the Methods. As expected in a population of individuals with common ancestry from East Africa, haplogroups L0-L4 were most commonly found. Because all subjects came from a common ancestral background, there was no difference in the distribution of haplogroups between the two groups see Table 2 .

\section{Mitochondria DNA sequence variation compared with the Cambridge Reference Sequence}

In the total group of 73 individuals tested, 4770 nucleotide variants were observed when compared with the revised Cambridge Reference Sequence resulting in an average frequency per individual of 3.97 variants per 1000 nucleotides (see Table 3). The median (IQR) number of variants/person for the entire mitochondrial sequence in the high lactate and normal lactate group was 57.5 (42-93) and 61 (45-90), respectively. The distribution of variants for the genes associated with oxidative phosphorylation was not statistically different between the two groups (see Fig. 1, left panel). Most of the variants occurred in more than one individual, and thus the number of unique variants identified in the entire cohort was 665 variants. The majority of these (565/665) were synonymous variants, while the remaining variants were non-synonymous variants expected to result in missense changes at the amino acid level. In-frame indels and lossof-function (nonsense, frameshift) variants were not identified.

\section{Non-synonymous variants within oxidative phosphorylation genes}

Although most variants resulted in synonymous coding, non-synonymous variants in the regions between nucleotide 3307 and 15,887 that code for 13 genes responsible for oxidative phosphorylation were the main focus of analysis as these variants are capable of creating mutations affecting enzyme function. Nonsynonymous variants to the revised Cambridge Reference Sequence were identified using the MSeqDR annotations. When combining the results for all oxidative phosphorylation genes, the frequency of nonsynonymous variants per person was not different
Table 3 Mitochondrial gene variants in 73 children with severe malaria anaemia

\begin{tabular}{|c|c|c|c|}
\hline & $\begin{array}{l}\text { Any variant } \\
\text { (full } \\
\text { sequence) }\end{array}$ & $\begin{array}{l}\text { Any variant } \\
\text { (oxphos } \\
\text { genes) }\end{array}$ & $\begin{array}{l}\text { Non- } \\
\text { synonymous } \\
\text { (oxphos genes) }\end{array}$ \\
\hline \multicolumn{4}{|c|}{ Number of variants found } \\
\hline All subjects, $n=73$ & 4770 & 2640 & 722 \\
\hline $\begin{array}{l}\text { High lactate, } \\
n=36\end{array}$ & 2365 & 1315 & 373 \\
\hline $\begin{array}{l}\text { Normal lactate, } \\
n=37\end{array}$ & 2405 & 1325 & 349 \\
\hline \multicolumn{4}{|c|}{ Median (IQR) variants/patient } \\
\hline All subjects, $n=73$ & $60(42-91)$ & $31(24-51)$ & $9(7-13)$ \\
\hline $\begin{array}{l}\text { High lactate, } \\
\mathrm{n}=36\end{array}$ & $57.5(42-93)$ & $31(24-51)$ & $9.5(8.5-13)$ \\
\hline $\begin{array}{l}\text { Normal lactate, } \\
n=37\end{array}$ & $61(45-90)$ & $31(27-50)$ & $9(7-12)$ \\
\hline \multicolumn{4}{|c|}{ Average (SD) variants/1000 nucleotides } \\
\hline All subjects, $n=73$ & $3.97(1.42)$ & $3.19(1.16)$ & $0.87(0.30)$ \\
\hline $\begin{array}{l}\text { High lactate, } \\
\mathrm{n}=36\end{array}$ & $3.99(1.53)$ & $3.22(1.26)$ & $0.91(0.33)$ \\
\hline $\begin{array}{l}\text { Normal lactate, } \\
n=37\end{array}$ & $3.95(1.32)$ & $3.16(1.07)$ & $0.83(0.26)$ \\
\hline
\end{tabular}

The number of variants, the median number (inter-quartile range, IQR) of variants per patient, and the average number of variants per patient per 1000 nucleotides is shown. The average number of non-synonymous variants per patient per 1000 nucleotides in genes of oxidative phosphorylation (oxphos) is not significantly different between the high lactate and normal lactate group, $\mathrm{p}=0.08$ (t-test)

between the groups with high and normal lactate levels (Fig. 1, right panel). Each of the oxidative phosphorylation genes was then analysed separately (see Table 4). Although the frequency of non-synonymous variants per person per 1000 nucleotides was slightly higher in the high lactate group for nearly every gene, the differences were not statistically significant. In both patient groups, non-synonymous variants per patient per 1000 nucleotides were found less frequently in ND4L/4 and were found more frequently in ATP8/6, ND3, and Cyto-b compared with the other oxidative phosphorylation genes. The number of individuals with one or more non-synonymous variants in each of the oxidative phosphorylation genes was also not different between the two groups.

Table 2 Mitochondrial haplogroup assignments in 73 Ugandan patients with severe malaria anaemia

\begin{tabular}{lcccccccc}
\hline & L0 & L1 & L2 & L3 & L4 & L5 & Other & Total \\
\hline High blood lactate & 9 & 4 & 6 & 14 & 3 & 0 & 36 \\
Normal blood lactate & 8 & 3 & 6 & 14 & 4 & 1 \\
Total & 17 & 7 & 12 & 28 & 7 & 1
\end{tabular}



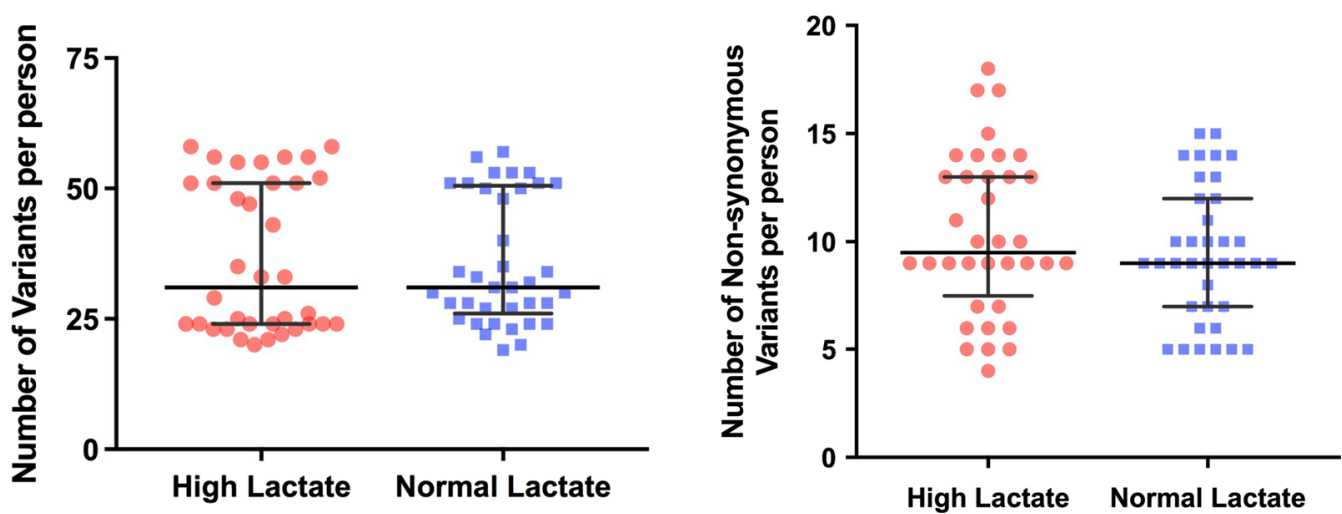

Fig. 1 Distribution of mitochondrial gene nucleotide variants in oxidative phosphorylation genes. The number of variants per person relative to the revised Cambridge Reference Sequence over the region of mitochondria encoding genes for oxidative phosphorylation is shown. Data from patients with high blood lactate levels $(n=36)$ and normal blood lactate levels $(n=37)$ are shown. Variants found at locations outside the coding sequence for the oxidative phosphorylation proteins are not included. Left panel shows results for any form of sequence variant. Right panel shows non-synonymous variants only. Horizontal bars are medians and inter-quartile ranges

Table 4 Non-synonymous variants per person compared with the Cambridge Reference Sequence

\begin{tabular}{|c|c|c|c|c|c|c|c|c|}
\hline & \multicolumn{2}{|l|}{ All subjects $n=73$} & \multicolumn{3}{|c|}{ High lactate $n=36$} & \multicolumn{3}{|c|}{ Normal lactate $n=37$} \\
\hline & Variants/person & $\begin{array}{l}\text { Variants/ } \\
\text { person/1000 } \\
\text { nts }\end{array}$ & Variants/person & $\begin{array}{l}\text { Variants/ } \\
\text { person/1000 } \\
\text { nts }\end{array}$ & $\begin{array}{l}\text { Number } \\
\text { of individuals }\end{array}$ & Variants/person & $\begin{array}{l}\text { Variants/ } \\
\text { person/1000 } \\
\text { nts }\end{array}$ & $\begin{array}{l}\text { Number } \\
\text { of individuals }\end{array}$ \\
\hline ND1 & 0.21 & 0.21 & 0.25 & 0.26 & 8 & 0.16 & 0.17 & 6 \\
\hline ND2 & 0.49 & 0.47 & 0.56 & 0.53 & 12 & 0.43 & 0.42 & 11 \\
\hline $\mathrm{COI}$ & 0.60 & 0.39 & 0.61 & 0.40 & 17 & 0.59 & 0.39 & 13 \\
\hline COII & 0.11 & 0.16 & 0.14 & 0.20 & 4 & 0.08 & 0.12 & 3 \\
\hline ATP8/6 & 2.44 & 2.90 & 2.42 & 2.87 & 36 & 2.46 & 2.92 & 37 \\
\hline COIII & 0.15 & 0.19 & 0.17 & 0.21 & 6 & 0.14 & 0.17 & 6 \\
\hline ND3 & 1.23 & 3.56 & 1.33 & 3.85 & 36 & 1.14 & 3.28 & 24 \\
\hline ND4L/4 & 0.18 & 0.11 & 0.19 & 0.12 & 7 & 0.16 & 0.10 & 4 \\
\hline ND5 & 1.38 & 0.76 & 1.56 & 0.86 & 25 & 1.22 & 0.67 & 24 \\
\hline ND6 & 0.14 & 0.26 & 0.17 & 0.32 & 5 & 0.11 & 0.21 & 4 \\
\hline Cyto-b & 2.96 & 2.59 & 2.97 & 2.60 & 36 & 2.95 & 2.58 & 37 \\
\hline Total & 9.89 & 0.87 & 10.36 & 0.91 & 36 & 9.43 & 0.83 & 37 \\
\hline
\end{tabular}

The average number of non-synonymous variants per person and the average number of variants per person per 1000 nucleotides (nts) is shown. The number of individuals with at least one non-synonymous variant is shown

Non-synonymous variants when present were seen in as few as $0.3 \%$ to as many as $100 \%$ of individuals depending on the nucleotide position. The distribution of individual non-synonymous variants in the study population is shown in Fig. 2.

\section{Non-synonymous variants predicted to represent a disruptive variant}

Because non-synonymous variants are predicted to result in different degrees of disruption of the final protein, the analysis further focused on those variants predicted to be most disruptive to the oxidative phosphorylation polypeptides. Four different software applications designed to predict disruptive variants were used: MToolBox [8, 9], PolyPhen2 [10], SIFT [11], and Mutation Taster [12]. Of the 100 different non-synonymous variants found within genes coding for oxidative phosphorylation, the 15 variants with the highest MToolBox disruption scores are shown in Table 5. Half of these occurred within genes coding for $\mathrm{NADH}$ dehydrogenase subunits (ND genes). When the data for the ND genes were combined in post hoc analysis, the frequency of disruptive variants at the 


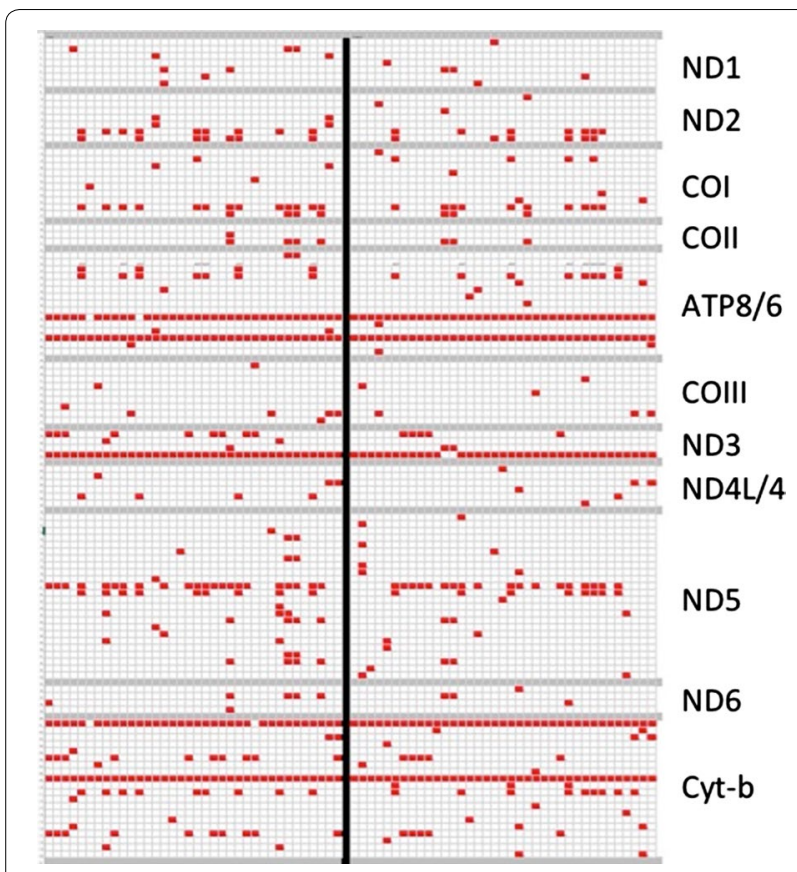

Fig. 2 Non-synonymous variants in oxidative phosphorylation genes among patients with and without elevated blood lactate levels. Patients with high blood lactate $(n=36)$ are on the left of the solid black line; patients with normal lactate levels $(n=37)$ are on the right. Each patient is represented by a column and each variant location by a row. Non-synonymous variants are shown as red squares. To simplify the figure, nucleotide positions (rows) without variants are not shown

seven ND locations was 0.087 in the high lactate group and 0.046 in the normal lactate group. Although this difference was not statistically significant $(\mathrm{p}=0.052$, Fisher's test) it suggests that disruptive variants of ND genes may be a target area for future study.

\section{Discussion}

Plasmodium falciparum malaria is a highly lethal and common disease of children worldwide. Lactic acidosis is a well-established complication of malaria infection and is associated with an increased risk for fatal outcome [6]. While lactic acidosis is present in approximately half of those with SMA, individual patients exhibit different levels of lactic acidosis despite the same degree of anaemia and many patients tolerate severe anaemia without developing elevated blood lactate levels $[3,5,6]$. The extent to which such differences are related to mitochondrial gene polymorphisms is unknown and was the focus of this study.

Malaria exerts a strong evolutionary selection pressure and is known to be associated with a large number of nuclear gene adaptations [4]. Although malaria infection has been associated with host mitochondrial pathology [13], previous studies exploring an association between severe malaria syndromes and human mitochondria DNA sequence variations were not identified. In this study, mitochondrial gene sequences between a cohort of children with or without lactic acidosis in the setting of SMA were compared for the first time. The two patient groups were well matched for ethnic background, and patients lived in close proximity to the hospital. The majority of mothers belonged to just three similar tribal affiliations. Mitochondrial haplotype analysis was balanced in the two groups and reflected haplogroups expected in an East African population $[14,15]$. Given the genetic heritage of the participants, a large number of synonymous and non-synonymous genetic variants were found when compared with the Cambridge Reference Sequence. These variants occurred throughout the mitochondrial genome.

The hypothesis of this study was that mitochondrial gene sequences might differ between those individuals who developed high blood lactate levels at the time of severe anaemia and those who did not. In particular, mitochondrial genes directly involved in oxidative phosphorylation were analyzed. Although the comparisons did not reach statistical significance for the sample size tested, the average number of non-synonymous variants per individual, the number of individuals with non-synonymous variants, and the occurrence of variants predicted to cause protein disruption were all higher in the group with elevated blood lactate levels. In particular, non-synonymous variants predictive to disrupt at least one member of the NADH dehydrogenase gene cluster were twice as common in children with high lactates, a finding that did not quite reach statistical significance $(p=0.052)$. Future studies with a larger sample size are needed to verify whether or not NADH dehydrogenase mutations might contribute to intolerance to severe anaemia.

The findings of this study suggest that the determinants of high blood lactate levels in patients with SMA may be related either to gene polymorphisms outside the mitochondrial genome or to other factors. Because the great majority of mitochondrial proteins are encoded by nuclear genes [16], variations in those genes might influence mitochondrial function. Alternatively, variation in other nuclear genes such as those of the glycolytic pathway, the Krebs cycle, or those affecting NAD+/NADH levels may influence blood lactate levels during severe anaemia. In addition, lactic acidosis in SMA may also be related to factors unrelated to host genetics such as microvascular physiology, nutritional status, or duration of illness. Understanding the fundamental drivers for life-threatening lactic acidosis in malaria awaits further research. 


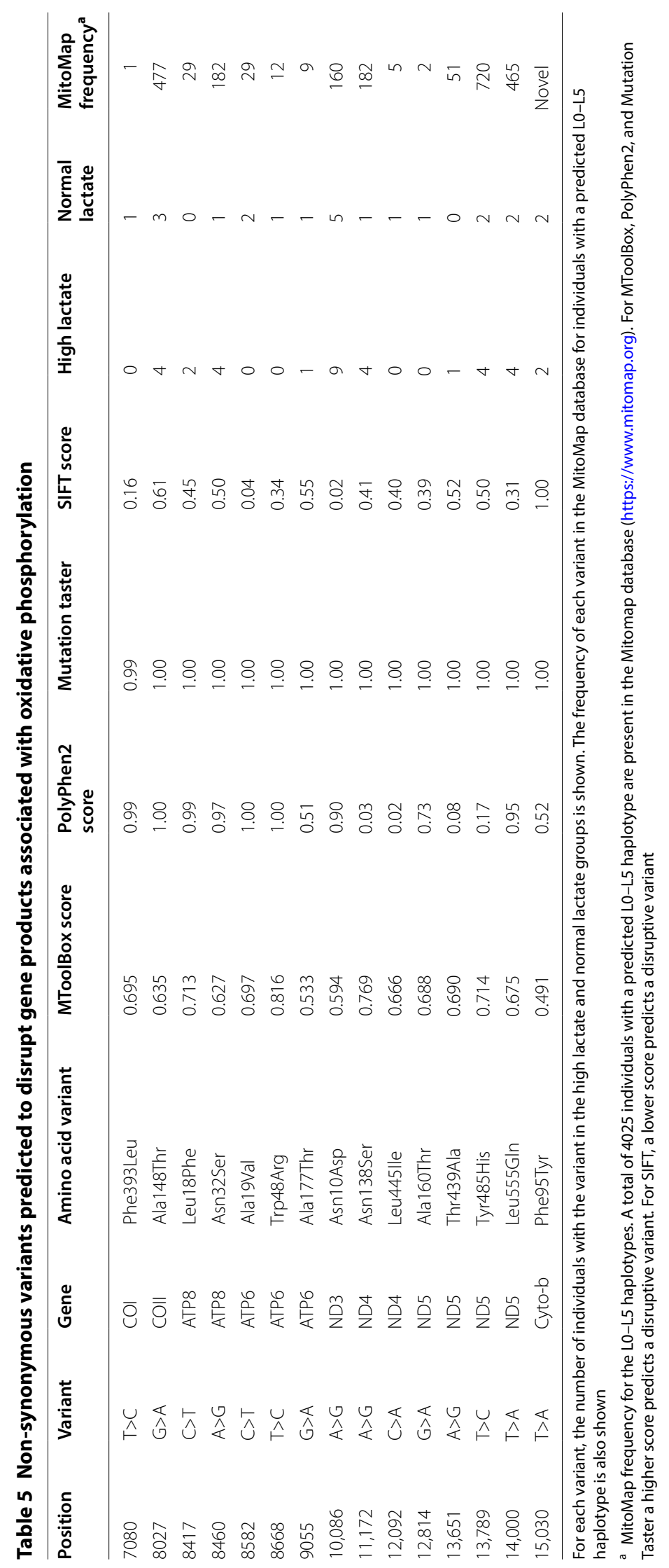


This study has both strengths and weaknesses. The comparison groups were homogeneous and well matched for clinical features including age, gender, and the degree of anaemia and haemoglobin oxygen saturation. In addition, all subjects were documented to be negative for the sickle haemoglobin gene which would be the most likely confounding variable to affect comparisons. The method of sample preparation avoided confounding from parasite mitochondrial DNA. However, the two groups were not compared for variations in nuclear genes that affect mitochondrial function, nor were the groups compared for the number of mitochondria present in tissues expected to produce the greatest amount of lactate. In addition, the number of individuals tested was insufficient to detect low frequency differences in mitochondrial gene sequences between the comparison groups.

\section{Conclusion}

In this prospective case-control investigation of 73 Ugandan children with severe anaemia and P. falciparum malaria, mitochondria sequence variations were observed to occur in approximately 4/1000 nucleotides compared with the Cambridge Reference Sequence. However, within the constraints of the sample size tested, no statistically significant difference in mitochondrial DNA sequence variation was observed when comparing those with and without lactic acidosis. Increased blood lactate, an important marker for decreased survival in SMA, does not appear to be strongly associated with a high prevalence host mitochondrial DNA sequence variation.

\section{Abbreviation}

SMA: severe malaria anaemia.

\begin{abstract}
Authors' contributions
CF performed DNA sequencing and sequence analysis and participated in manuscript preparation. CC-G participated in study design, funding, clinical data collection, and manuscript preparation. AD enrolled patients, collected samples and clinical data, and participated in manuscript preparation.CM enrolled patients, collected samples and clinical data, and participated in manuscript preparation. HS was responsible for statistical review. SA participated in study design, DNA testing and analysis, and manuscript preparation. WD participated in study design, funding, clinical data collection, data analysis, and manuscript preparation. All authors read and approved the final manuscript.
\end{abstract}

\section{Author details \\ ${ }^{1}$ Translational Genomics Core Laboratory, Partners Personalized Medicine, Boston, MA, USA. ${ }^{2}$ Department of Pathobiology and Laboratory Medicine, University of Toronto, Toronto, Canada. ${ }^{3}$ Department of Pediatrics and Child- health, Makerere University, Kampala, Uganda. ${ }^{4}$ Department of Medicine, Makerere University, Kampala, Uganda. ${ }^{5}$ Department of Pathology and Medi- cine, Massachusetts General Hospital, Boston, MA, USA.}

\section{Acknowledgements}

The molecular methods (DNA extraction, library preparation and sequencing of mitochondrial genomes) was performed at the Translational Genomics Core at Partners HealthCare Personalized Medicine. We would like to thank
Ashley Blau, MS and the team at the Translational Genomics Core for their contributions to this effort.

\section{Competing interests}

The authors declare that they have no competing interests.

Availability of data and materials

Sequence data are available from the authors upon request.

Consent for publication

Not applicable.

\section{Ethics approval and consent to participate}

This study was approved by the Partners Healthcare Research Ethics review committee, by the Makerere University College of Medicine research review committee, and by the Uganda National Council of Science and Technology.

\section{Funding}

The study was funded by Grants from the Heart Lung and Blood Institute of the NIH (NIH 1R21HL109518-01A1) to WD; the International Society of Blood Transfusion to CC-G; the National Blood Foundation to CC-G; and the Robert and Evelyn Luick Fund to WD. Funding agencies had no role in the design, analysis, interpretation, or manuscript preparation.

\section{Publisher's Note}

Springer Nature remains neutral with regard to jurisdictional claims in published maps and institutional affiliations.

Received: 5 April 2018 Accepted: 8 December 2018

Published online: 13 December 2018

\section{References}

1. Gething PW, Casey DC, Weiss DJ, Bisanzio D, Bhatt S, Cameron E, et al. Mapping Plasmodium falciparum mortality in Africa between 1990 and 2015. N Engl J Med. 2016;375:2435-45.

2. Mangano VD, Modiano D. An evolutionary perspective of how infection drives human genome diversity: the case of malaria. Curr Opin Immunol. 2014;30:39-47.

3. Cserti-Gazdewich C, Dhabangi A, Musoke C, Ssewanyana I, Ddungu H, Nakiboneka- Ssenabulya D, et al. Cytoadherence in paediatric malaria: ABO blood group, CD36, and ICAM-1 expression and severe Plasmodium falciparum infection. Br J Haematol. 2012;159:223-36.

4. Kwiatkowski D. How malaria has affected the human genome and what human genetics can teach us about malaria. Am J Hum Genet. 2005;77:171-90.

5. Dhabangi A, Ainomugisha B, Cserti-Gazdewich C, Ndungu H, Kyeyune $D$, Musisi E, et al. Effect of transfusion of red blood cells with longer vs shorter storage duration on elevated blood lactate levels in children with severe anemia: the TOTAL randomized trial. JAMA. 2015;314:2514-23.

6. Cserti-Gazdewich C, Dhabangi A, Musoke C, Ssewanyana I, Ddungu H, Nakiboneka- Ssenabulya D, et al. Inter-relationships of cardinal features and outcomes of symptomatic pediatric Plasmodium falciparum malaria in 1933 children in Kampala, Uganda. Am J Trop Med Hyg. 2013;88:747-56.

7. https://support.illumina.com/content/dam/illumina-support/docum ents/documentation/chemistry_documentation/samplepreps_legacy/ human-mtdna-genome-guide-15037958-01.pdf. Accessed 4 Apr 2018.

8. Calabrese C, Simone D, Diroma MA, Santorsola M, Gutta C, Gasparre G, et al. MToolBox: a highly automated pipeline for heteroplasmy annotation and prioritization analysis of human mitochondrial variants in highthroughput sequencing. Bioinformatics. 2014;30:3115-7.

9. Santorsola M, Calabrese C, Girolimetti G, Diroma MA, Gasparre G, Attimonelli M. A multi-parametric workflow for the prioritization of mitochondrial DNA variants of clinical interest. Hum Genet. 2016;135:121-36.

10. Adzhubei IV, Schmidt S, Peshkin L, Ramensky VE, Gerasimova A, Bork P, et al. A method and server for predicting damaging missense mutations. Nat Methods. 2010;7:248-9. 
11. Kumar P, Henikoff S, Ng PC. Predicting the effects of coding non-synonymous variants on protein function using the SIFT algorithm. Nat Protoc. 2009:4:1073-81.

12. Schwarz JM, Rodelsperger C, Schuelke M, Seelow D. MutationTaster evaluates disease-causing potential of sequence alterations. Nat Methods. 2010;7:576-86.

13. Dey S, Guha M, Alam A, Goyal M, Bindu S, Pal C, et al. Malarial infection develops mitochondrial pathology and mitochondrial oxidative stress to promote hepatocyte apoptosis. Free Radic Biol Med. 2009:46:271-81.

14. Cohen T, Levin L, Mishmar D. Ancient Out-of-Africa mitochondrial DNA variants associate with distinct mitochondrial gene expression patterns. PLoS Genet. 2016;12:e1006497.
15. Silva M, Alshamali F, Silva P, Carrilho C, Mandlate F, Jesus Trovoada M, et al. 60,000 years of interactions between Central and Eastern Africa documented by major African mitochondrial haplogroup L2. Sci Rep. 2015;5:12526

16. Cotter D, Pumima G, Fahy E, Subramaniam S. MitoProteome: mitochondrial protein sequence database and annotation system. Nucleic Acids Res. 2004;32:D463-7.
Ready to submit your research? Choose BMC and benefit from:

- fast, convenient online submission

- thorough peer review by experienced researchers in your field

- rapid publication on acceptance

- support for research data, including large and complex data types

- gold Open Access which fosters wider collaboration and increased citations

- maximum visibility for your research: over $100 \mathrm{M}$ website views per year

At BMC, research is always in progress.

Learn more biomedcentral.com/submissions 